\title{
Cross-Sectional Study on Awareness and Knowledge of COVID-19 Among Senior pharmacy Students
}

\author{
Marwa S. Hamza ${ }^{1,2}$ (D) Osama A. Badary ${ }^{1,2} \cdot$ Mohamed M. Elmazar ${ }^{2,3}$
}

Published online: 15 June 2020

(c) Springer Science+Business Media, LLC, part of Springer Nature 2020

\begin{abstract}
Extraordinary actions have been implemented in an effort to control the rapid spread of the ongoing COVID-19 epidemic in Egypt. People's adherence to control measures is influenced by their knowledge, attitudes and practices towards the disease. Therefore, in the present study we assessed pharmacy senior students' knowledge, attitudes and practices towards the COVID-19 pandemic. An online questionnaire was created and it consisted of 12 questions testing their knowledge about COVID-19 clinical characteristics, transmission routes and prevention and control steps. Among senior pharmacy students $(\mathrm{n}=238), 70 \%$ were females and $63 \%$ were living in greater Cairo. Their main source of information included social media (70\%), published articles (48\%) and television (48\%). The overall correct knowledge score was $83 \%$. Most of the students displayed a good COVID-19 knowledge level ( $72.5 \%$ of the students). The students were least informed when trying to answer questions about hyper-coagulation, as a major cause for death in patients with severe COVID-19, and about the timings on the necessity to wear masks. Assessment of students' attitudes and practices towards COVID-19 reflected that $87 \%$ of them were confident that health care teams and scientists could win the fight against the virus. In addition, $72 \%$ of students agreed that COVID-19 will be controlled successfully. The greater the students' knowledge, the more confident they felt that COVID-19 will be controlled successfully (OR 2.2, 95\% confidence interval [CI] 1.03-4.72). Good behavioral practice towards COVID-19 control was confirmed when $87 \%$ of students answered that they didn't go out to any crowded place. Females were 3.6 times (95\% confidence interval [CI] 1.03-3.11) more likely to avoid going out than males. Bad behavioral practice became evident when approximately $50 \%$ of students admitted that they did not wear masks when they left their house. Therefore, more efforts should be taken to protect future pharmacists from this pandemic.
\end{abstract}

Keywords COVID-19 $\cdot$ Knowledge $\cdot$ Attitude $\cdot$ Practice $\cdot$ Egypt

\section{Introduction}

An epidemic, triggered by the new coronavirus SARSCoV-2, has recently become the focus of the scientific community attention [1]. COVID-19 represents the illness

Marwa S. Hamza

marwa.hamza@bue.edu.eg

1 Clinical Pharmacy Practice Department, Faculty of Pharmacy, The British University in Egypt, P.O. Box 43, El-Sherouk City, Cairo 11837, Egypt

2 The Center for Drug Research and Development (CDRD), Faculty of Pharmacy, The British University in Egypt, P.O. Box 43, El-Sherouk City, Cairo 11837, Egypt

3 Pharmacology and Biochemistry Department, Faculty of Pharmacy, The British University in Egypt, P.O. Box 43, El-Sherouk City, Cairo 11837, Egypt generated by this virus. Its clinical presentation ranges from being an asymptomatic infection to developing into a severe disease with high mortality rate [2]. At the time of writing (May 16, 2020), over 4,425,485 COVID-19 cases and 302,059 death have been registered worldwide [3]. In particular, in Egypt, there are 11,228 positive individuals and 592 death cases [4]. Now, COVID-19 has been classified as a pandemic by the World Health Organization. It is both a highly contagious and a life-threatening disease [3]. Its reproduction ratio (RR) is defined as the expected number of cases generated by a single infected individual within a susceptible population [5]. It varies between 2.4 and 2.7 [6]. These values are high compared to influenza $(\mathrm{RR}=1.3-1.8)$ [7].

Currently, a fight is being undertaken against the COVID-19 pandemic. Adherence to control and prevention steps is paramount for ensuring a complete success 
over COVID-19. Lessons were learned from the 2003 SARS outbreak. These indicate that fear exists within the population awareness and attitude to infectious diseases. This factor may hinder further efforts to prevent the virus spread. Therefore, it is crucial to understand COVID-19 public's awareness at this critical stage to facilitate pandemic management $[8,9]$.

As members of the health care team, pharmacists play an essential role in patient care. This includes contributing to the decision-making process within the multidisciplinary health care team, taking responsibility for medication administration and assessment of patient care [10]. Moreover, together with physicians, pharmacists' knowledge, attitude and practices are crucial to prevent and control the disease [11]. Health care teams include pharmacists are responsible for providing knowledge, delivering good quality management and protecting individuals from illness during epidemic prevalence period. Therefore, pharmacy students' education should include training in precautionary measures, effective treatment and follow-up. This is critical together with their behavior in these fields [12]. Normally, training in disaster medicine occupy a very small place in regular medical curricula worldwide [13]. Therefore, the present study was carried out to evaluate knowledge about COVID-19 as an attempt to identify senior students' degree of awareness and its effects. Furthermore, this study objective aims to contribute in developing a fit for purpose education program that creates awareness among future practitioners. To the best of the authors' knowledge, this is the first study directly exploring senior pharmacy students' knowledge, attitudes and practice regarding this topic.

\section{Subjects and Methods}

\section{Study Design and Population}

This is an institutional based cross sectional study conducted in Faculty of Pharmacy at the British University in Egypt during the period from April 28, 2020 to April 30, 2020. The targeted population included students who had completed $\geq 85 \%$ of the credit-hours required for graduation.

\section{Sample Size}

The sample size was calculated by using the online sample size calculator RaoSoft ${ }^{\circledR}$. Based on an estimated population of 470 students in the final years (year 4 and 5) and on the anticipated response of $50 \%$, the minimum required sample size was 204 participants with a confidence level of $95 \%$ and a $5 \%$ margin of error.

\section{Study Instrument}

A pre-validated questionnaire [14] was modified after a comprehensive literature search and based on the most recent available information from the World Health Organization, the Center for Disease Control and Prevention (USA) and the Egypt Ministry of Health. The initial draft was sent to a group of experts, chosen according to their experience and expertise in related fields, to appraise the questions in terms of relativity, simplicity and importance. Then, the questionnaire was sent by email, through Survey Monkey Software ${ }^{\circledR}$. A pilot study was conducted on 10 subjects, to test the questionnaire validity. Following a group discussion, the questionnaire was completed. The data from the pilot study was removed from the final analysis.

The questionnaire included two sections: demographics, and knowledge, attitudes and practices questions (KAP). Demographic variables included age, gender and place of current residence (Cairo, other governorates in Egypt). The first part of the KAP represented the knowledge investigation, consisting of 12 questions (Fig. 1): 4 questions were about COVID-19 clinical presentations (K1-K4), 3 asked about transmission routes (K5-K7), and the following 5 questions quizzed on prevention and control (K8K12). These questions were answered on a true/false basis, including an additional "I don't know" option. One point was assigned to a correct response, while no points were given for an incorrect/unknown answer. The total score ranged from 0 to 12 , with a higher score suggesting better COVID-19 awareness. In the pilot sample, the knowledge questionnaire Cronbach's alpha coefficient was 0.73 , indicating reasonable internal consistency [15].

Attitudes towards COVID-19 were appraised with 2 questions (A1-A2, Fig. 2) that asked about the level of agreement on the COVID-19 control implementations and the confidence perception for winning the war against this coronavirus. The assessment of students practices was performed through 4 questions (P1-P4, Fig. 2), that investigated their actions towards going to a crowded place, wearing masks when going out in recent days, maintaining social distance between others and if they agree that patients should disclose their exposure to COVID-19. Finally, one last question was added to inquire about the students' source of COVID-19 information.

\section{Ethical Considerations}

Before study start-up, permission was taken from the ethics committee of the Faculty of Pharmacy at the British University in Egypt. The participants were briefed about 


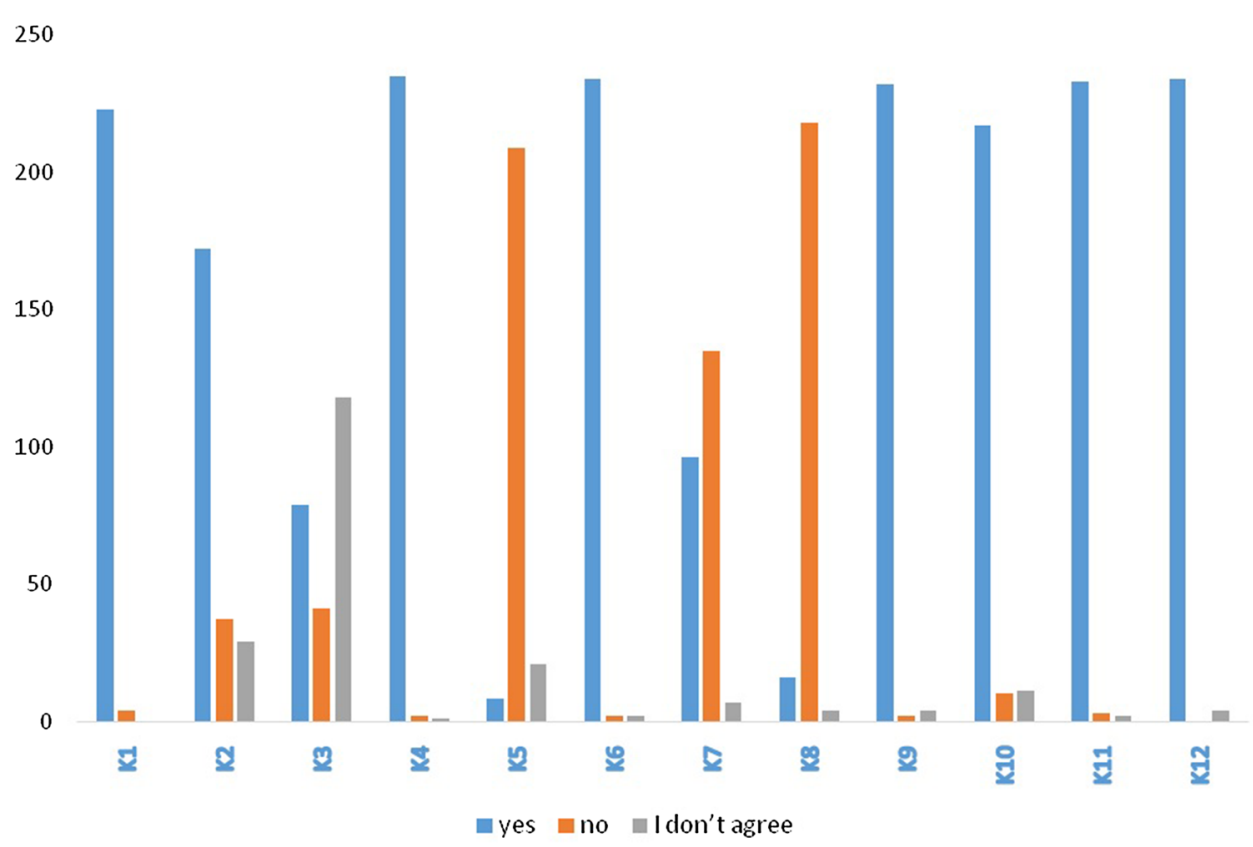

Fig. 1 Knowledge of students towards COVID-19. K1. The main clinical symptoms of COVID-19 are fever, tiredness, and dry cough, K2 Some patients infected with the COVID-19 virus may suffer from aches and pains, nasal congestion, runny nose, sore throat or diarrhea, K3. Coagulation dysfunction is one of the major causes for death in patients with severe COVID-19, K4. Older persons and persons with pre-existing medical conditions (such as high blood pressure, heart disease, lung disease, cancer or diabetes) appear to develop serious illness with COVID-19 more often than others, K.5 People with COVID-19 will not transmit the virus to others when fever is not present, K6. COVID-19 can spread from person to person through small droplets from the nose or mouth which are spread when a person with COVID-19 coughs or exhales, K7. Only wear a mask if you are ill

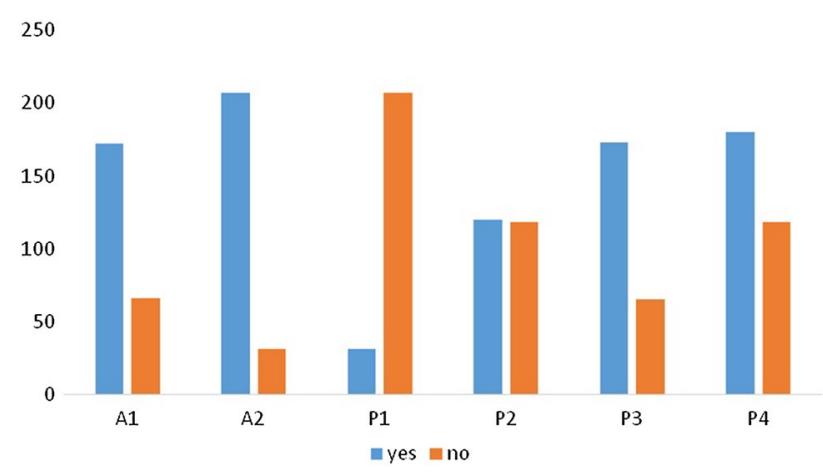

Fig. 2 Attitudes \& practice of students towards COVID-19. A1. Do you agree that COVID-19 will be successfully controlled? A2. Are you confident that the health care team and scientists can win the fight against the COVID-19 virus? P1. In recent days, have you gone to any crowded place? P2. In recent days, if you left your house, have you worn a mask when leaving home? P3. In recent days, did you keep your social distance at least $2 \mathrm{~m}$ from other people? P4. Do you agree that patients should disclose their exposure to COVID-19? with COVID-19 symptoms or looking after someone who may have COVID-19, K8. It is not necessary for children and young adults to take measures to prevent the infection by the COVID-19 virus, K9. To prevent the infection by COVID-19, individuals should avoid going to crowded places such as public transportation, K10. COVID19 is caused by a virus, so antibiotics do not work. Antibiotics should not be used as a means of prevention or treatment of COVID-19. They should only be used as directed by a physician to treat a bacterial infection, K11. Isolating and treating people with COVID-19 infections are effective means of minimizing viral spread, K12. People who have contact with someone who has COVID-19 infection should be isolated in a proper place immediately. The observation period is usually 14 days

the study rationale and were reassured about the confidentiality safeguards for their personal information and responses. The answered survey submission was considered as consent for study participation. Respondents' participation was completely consensual, anonymous and voluntary.

\section{Statistical Analysis}

The completed questionnaires data were analyzed using the Statistical Package for Social Science, version 21.0 (SPSS 20.0, Chicago, IL). The significance level was set at a value of $p<0.05$ for all analyses. Initially, all information gathered through the questionnaire was coded into variables. In the pilot study, the Cronbach's alpha coefficient test was used to measure internal consistency. Data normality was tested using the Kolmogorov-Smirnov test. Descriptive and inferential statistics, involving Chi-square test, Mann-Whitney U test, correlation and logistic regression, were used to analyze the results. 
In the present study, the scoring system and categorization were utilized to assess knowledge levels towards the COVID-19 pandemic. The questions determination was based on one point for every correct answer and zero points for false answers. By adding the results of all the knowledge questions in the survey, the participants mean knowledge scores (KSs) were determined. KS ranged from 0 to 12 , with the higher scores demonstrating an increased level of pandemic COVID-19 knowledge. Knowledge level categories were defined by an $80 \%$ cut-off point as any score above $80 \%$ is good and any score below $80 \%$ is poor [16].

\section{Results}

\section{Subjects' Characteristics}

During the course of this study, a total of 248 participants completed the devised online survey questions. After excluding 10 respondents who participated in the pilot study, the final sample consisted of 238 participant students. Among these, the average age (measured in years) was $22 \pm 1,166$ students (70\%) were females. When identifying the place of residence, 149 students $(63 \%)$ were living in greater Cairo while 89 (37\%) resided in other governorates (Table 1). With regards to the students' source of information, results disclosed that the main source of information was social media (70\%) followed by published articles (48\%) and television (48\%).

Table 1 Demographic profile of senior pharmacy students $(n=238)$

\begin{tabular}{lll}
\hline Socio-demographic characteristics & No & $(\%)$ \\
\hline Age (years)(mean \pm SD, range) & $22 \pm 1,19-26$ & \\
Gender & & \\
Male & 72 & 30 \\
Female & 166 & 70 \\
Place of current residence & & \\
Greater Cairo & 149 & 63 \\
Other governorates & 89 & 37 \\
Sources of information & & \\
Physicians & 72 & 30 \\
Pharmacists & 81 & 34 \\
Friends & 45 & 19 \\
Social media & 166 & 70 \\
Articles & 114 & 48 \\
TV & 114 & 48 \\
Knowledge score (mean \pm SD, range) & $10 \pm 1.2,4-12$ & \\
Good knowledge & 175 & 73.5 \\
Poor knowledge & 63 & 26.5 \\
\hline
\end{tabular}

\section{Characteristics of COVID-19 Related Knowledge}

In the present study, the main objective was to measure COVID-19 related knowledge of pharmacy senior students. The mean score of COVID-19 related knowledge was $83 \%$ (10 \pm 1.2 , ranging from 4 to 12$)$ (Table 1$)$. In particular, $72.5 \%$ of the students possessed a good knowledge level, while $23.5 \%$ displayed poor knowledge based on an $80 \%$ cut-off point [16]. The first 4 survey questions (K1-K4) measured students' knowledge towards COVID-19 clinical presentations. The highest correct answer rate (98\%) related to the COVID-19 related knowledge items identified by 2 questions; 'the main clinical symptoms of COVID-19 are fever, tiredness, and dry cough (True)' and 'older persons and persons with pre-existing medical conditions (such as high blood pressure, heart disease, lung disease, cancer or diabetes) appear to develop serious illness with COVID-19 more often than others (True)'(Fig. 1). However, the item with the lowest correct-answer rates was: 'coagulation dysfunction is one of the major causes for death in patients with severe COVID-19 (True)' (37\%) (Fig. 1). The answer 'I don't know' was selected by $12 \%$ of students for the question 'COVID-19 may cause aches and pains, nasal congestion, runny nose, sore throat or diarrhea in some patients' (Fig. 1). Then the following 3 questions (K5-K7) measured students' knowledge towards the transmission routes. In this case, the percentage of knowledgeable students is high. K.5: no transmission if fever is not present (88\%); K6: COVID-19 can spread from person to person through small droplets (98\%); and K7: wear a mask only if you are ill or looking after someone who may have COVID-19 (96\%). A similar result was achieved with the next 5 questions (K8-K12), which concerned assessing knowledge about COVID-19 prevention and control (Fig. 1). This study showed a significant relation between the knowledge score and published articles as source of information $(\mathrm{P}<0.05)$.

\section{Characteristics of COVID-19 Related Attitude and Practice}

The students' attitude was measured with 2 questions. The highest positive result was attained by the following COVID-19-related attitude item: 'Are you confident that the health care team and scientists can win the fight against the COVID-19 virus?' (87\% answered yes). However, the lowest positive attitude result corresponded to the following: 'Do you agree that COVID-19 will be successfully controlled?' (only $72 \%$ answered yes) (Fig. 2).

The highest performance rate was attained by the following COVID-19-related practice item: 'In recent days, have you gone to any crowded place?' ( $87 \%$ answered no). However, the item with the lowest performance rate was: 'In recent days, if you left your house, have you worn a 
mask when leaving home?' (50.5\% gave a negative answer) (Fig. 2). As expected, it was found that the COVID-19 practice for going out in crowded place was significantly different between males and females: females displayed a lower tendency to go out than males $(\mathrm{P}<0.05)$. In addition, the practice for keeping social distance between students was significantly different between males and females: females maintained social distance more successfully than males $(\mathrm{P}<0.05)$. It is worth mentioning that significant relations existed between the two practices and the students' source of information. In particular, the practice of wearing a mask when leaving home was directly correlated with using television as source of information $(\mathrm{P}<0.05)$. Furthermore, the practice of keeping social distance of at least $2 \mathrm{~m}$ from other people was directly associated with utilizing physicians' advice as source of information $(\mathrm{P}<0.05)$.

Moreover, the correlation coefficient, existing between the major knowledge, attitude and practice variables was analyzed. A weak positive correlation was observed between attitude and practice and this result appeared to be highly significant $(\mathrm{r}=0.2 ; \mathrm{p}<0.01)$. However, no correlation appeared to exist between knowledge and attitudes or knowledge and practice (Table 2).

Logistic regression analyses showed that students with a good knowledge score are 2.2 times more optimistic and agreed that COVID-19 will be successfully controlled (95\% confidence interval [CI] 1.03-4.72). Students who live in Cairo were 1.7 times more likely to maintain social distance of at least $2 \mathrm{~m}$ from other people (95\% confidence interval [CI] 0.9-3.2)., and 1.7 times more likely to agree that patients should disclose potential COVID-19 exposure, than

Table 2 Correlation between knowledge, attitude and practice

\begin{tabular}{lll}
\hline Variables & rho & P-value \\
\hline Knowledge \&c attitude & -0.058 & 0.37 \\
Knowledge \& practice & 0.006 & 0.891 \\
Attitude \& practice & 0.159 & $0.014^{*}$ \\
\hline
\end{tabular}

*Statistically significant at $\mathrm{p}<0.05$ students who live in other governorates in Egypt (95\% confidence interval [CI] 0.9-3.3). Moreover, female students were 3.6 times more likely to avoid going to any crowded place than male students (95\% confidence interval [CI] 1.5-8.6), and 2.3 times more likely to keep their social distance of at least $2 \mathrm{~m}$ from other people than male students (95\% confidence interval [CI] 1.2-4.4) (Table 3).

\section{Discussion}

The coronavirus disease 2019 (COVID-19) is an emerging contagious respiratory disease caused by a novel coronavirus. It was first detected in December 2019 in Wuhan, China [17]. Emerging infectious diseases like COVID-19 can occur anywhere in the world. By being part of the health care team, pharmacists can be exposed to the risk of infection [18]. Here, we present the results of a questionnaire study about the knowledge, attitudes and practices of Egyptian senior pharmacy students towards the COVD-19 disease.

In the present study, one of the most significant findings was that the highest main source of COVID-19 information among senior students was social media, television and published articles. The results strongly support similar findings in which the main source of Middle East respiratory Syndrome (MERS) information was reported to be the internet and social media [19]. This clearly indicates that senior pharmacy students are relying consistently on the internet, social media and online information as the principal ways to obtain information, compared with friends and other resources. The Egyptian Ministry of Health and Population and the World Health Organization (WHO) have provided clusters with COVID-19 information through their websites and are recommending everyone to be aware of updates relating to knowledge about COVID-19 [3, 4]. Similarly, a previous study reported that internet was the main source of information about MERS-CoV [20]. Therefore, it is feasible to suggest that researches should urgently find ways to utilize the Internet to promote health, especially in emerging infectious diseases and pandemics. At present, the

Table 3 Significantly associated factors towards COVID-19 logistic regression analysis of odds ratio (OR) for attitudes and practices in relation to potential risk factors

\begin{tabular}{|c|c|c|c|c|c|c|}
\hline \multirow[t]{2}{*}{ Variable } & \multicolumn{2}{|l|}{ Attitude } & \multicolumn{4}{|l|}{ Practice } \\
\hline & A1 & $\mathrm{A} 2$ & $\mathrm{P} 1$ & $\mathrm{P} 2$ & P3 & $\mathrm{P} 4$ \\
\hline Knowledge category & $2.2^{*}(1.03-4.72)$ & $0.9(0.34-2.04)$ & $1.2(0.4-3.2)$ & $1.2(0.7-2.3)$ & $0.8(0.4-1.6)$ & $0.8(0.4-1.7)$ \\
\hline Gender & $1.5(0.81-2.95)$ & $0.8(0.32-1.99)$ & $3.6^{*}(1.5-8.6)$ & $1.2(0.6-2.2)$ & $2.3^{*}(1.2-4.4)$ & $0.9(0.4-1.8)$ \\
\hline $\operatorname{Age}^{\mathrm{a}}$ & $1.1(0.8-1.49)$ & $0.7(0.50-1.17)$ & $0.9(0.6-1.3)$ & $1.1(0.8-1.4)$ & $0.9(0.7-1.2)$ & $0.9(0.6-1.3)$ \\
\hline Residence & $1.2(0.66-2.24)$ & $1.3(0.59-3.00)$ & $0.8(0.3-2.03)$ & $0.9(0.5-1.5)$ & $1.7^{*}(0.9-3.2)$ & $1.7^{*}(0.9-3.3)$ \\
\hline
\end{tabular}

*Statistically significant at $\mathrm{p}<0.05$

${ }^{\text {a }}$ Scored as continuous variable 
quality of the information available on the Internet is insufficient and needs to be revised. Furthermore, a very small percentage of participants reported that healthcare professionals, either physicians or pharmacists, were their primary source of information. This scenario illustrates the lack of healthcare team engagement in increasing the public awareness regarding different aspects of COVID-19.

The present study showed a significant relation existing between the knowledge score and published articles as source of information $(\mathrm{P}<0.05)$. That may be due to the role played by the WHO and the Egyptian Ministry of Health and Population in providing cluster with COVID-19 information through their websites. Therefore, these organizations recommend everyone to be aware of updates about COVID-19.

As mentioned earlier, 238 senior pharmacy students participated in the present study. The overall mean knowledge scored among students was $83 \%(10 \pm 1.2)$. Most of the students possessed a good COVID-19 knowledge level (72.5\% of the students), possibly owing to the fact that the Egyptian Ministry of Health and Population (MOHP) and the World Health Organization (WHO) are providing a significant amount of COVID-19 information through their websites [3, 4]. The present study results showed that $98 \%$ of participants were knowledgeable about the main clinical symptoms of COVID-19. In addition, the same percentage of students knew that older persons and persons with preexisting medical conditions were more liable to develop serious illness with COVID-19 than others. These findings confirm observations from previous studies, which reported that the majority of participants believed that the disease is more dangerous for the elderly and for those suffering from chronic diseases [21, 22].

The students were least knowledgeable towards the question of coagulation dysfunction being one of the major death causes in patients with severe COVID-19. Suitable remedial actions should be taken since vascular, venous thromboembolism and arterial thrombosis risks have been documented in severe and critically ill COVID-19 patients $[23,24]$. A perceived lack of information regarding some of the COVID-19 clinical presentation or preventive measures highlights the requirement for the inclusion of more training, seminars in their courses. This raises concerns regarding the ability of newly-graduated pharmacists to deal with the COVID-19 pandemic disease, or any other potential similar epidemic events. Also, the students were least knowledgeable about when to wear masks. Masks and sanitizing products use has evolved since the beginning of the coronavirus pandemic, resulting in their rapid depletion. Lack of appropriate security measures represents a major concern for health workers. This is a cause of concern in a highly populated country such as Egypt, where the public may experience anxiety and even panic feelings, because basic protection measures are unavailable [25].
Knowledge is a prerequisite for establishing positive attitudes and promoting positive behaviors. Therefore, it makes sense to invest in individuals' cognition towards the infection. Regarding the senior students' attitude towards COVID-19, in the present study $72 \%$ of them agreed that COVID-19 will be successfully controlled and $87 \%$ were confident that health care teams and scientists can win the fight against the coronavirus. Interestingly, it was found that a greater students' knowledge relates to more confidence in COVID-19 becoming successfully controlled (OR 2.2, 95\% confidence interval [CI] 1.03-4.72).

Correlation between the students' attitude and practice indicates that their positive attitude is affecting their action towards COVID-19. This present study results showed that $87 \%$ of participants did not go to any crowded place, $76 \%$ of the students agreed that patients should disclose their exposure to COVID-19 and $73 \%$ of the students kept the social distance of at least $2 \mathrm{~m}$ from other people. However, the students showed bad practice when using masks as a protective measure (approximately 50\%). That was directly related to their lower level of knowledge towards the importance of wearing masks $(\mathrm{P}<0.05)$. That is supported by findings from a previous study, which reported that only about $35 \%$ of the participants were willing to wear face masks [21].

Interestingly, it was reported that females preferred not to go to any crowded place more than male students (OR 3.6, 95\% confidence interval [CI] 1.5-8.6). In addition, females preferred to keep the social distance more than male students (OR 2.3, 95\% confidence interval [CI] 1.2-4.4). Moreover, students who live in other governorates kept social distance and agreed that patients should disclose their exposure to COVID-19 rather than the students who live in the capital (OR 1.7, 95\% confidence interval [CI] 0.9-3.2).

It is noteworthy that a significant association existed between the practice of wearing masks when leaving home and using television as source of information $(\mathrm{P}<0.05)$. Likewise, an association existed between the practice of keeping the social distance at least $2 \mathrm{~m}$ from other people and utilizing physicians as a source of information $(\mathrm{P}<0.05)$. This encourages the public to avoid public meetings, including sports, ceremonies, meetings and school classes, in order to prevent a global transmission of coronavirus infection [26]. However, many people ignore the importance of maintaining social distance because of attitudinal issues.

\section{Conclusions and Recommendations}

It can be concluded that senior pharmacy students are generally knowledgeable about COVID-19. They lack knowledge about some of the clinical presentation of severe COVID19 infection such as hyper-coagulation. They also lack 
knowledge about the indication of wearing face masks. The present study constitutes the first attempt to examine knowledge and perceptions of future pharmacists in Egypt on pandemic diseases. Finally, the analysis of senior pharmacy students' knowledge and the factors affecting their attitudes and practices towards COVID-19 could provide a reference for preventing further spread of this disease.

It is recommended that strategies and education programs should be implemented to reduce the risk of infection among future pharmacists. Senior pharmacy students should acquire increased awareness regarding the seriousness and consequences of a COVID-19 infection.

\section{Limitations of the Study}

Some of the results of this study were compared with research on other new emerging infectious diseases similar to coronavirus, because no analyses have been conducted concerning pharmacy students or students in general.

Acknowledgements The researchers acknowledge our senior pharmacy students who took part in this study.

\section{Compliance with Ethical Standards}

Conflict of interest The authors declare that they do not have conflict of interests.

\section{References}

1. Fauci, A. S., \& Lane, H. C. (2020). Covid-19-navigating the uncharted. The New England Journal of Medicine, 382(13), 12681269. https://doi.org/10.1056/NEJMe2002387.

2. Carsetti, R., Rosado, M. M., Donnanno, S., et al. (2005). The loss of IgM memory B cells correlates with clinical disease in common variable immunodeficiency. The Journal of Allergy and Clinical Immunology, 115(2), 412-417. https://doi.org/10.1016/j. jaci.2004.10.048.

3. World Health Organization. Coronavirus Disease 2019 (COVID19) Situation Report-117. situation-reports/20200311-sitrep51-covid-19.pdf?sfvrsn=1ba62e57_10 2020. Retrieved 16 May 2020 from https://www.who.int/emergencies/diseases/novel-coron avirus-2019

4. Ministry of Health-Egypt. COVID-19 overview. 2020. Retrieved 16 May 2020 from https://www.care.gov.eg/EgyptCare/Index .aspx.

5. Fraser, C., Donnelly, C. A., Cauchemez, S., et al. (2009). Pandemic potential of a strain of influenza A (H1N1): Early findings. Science, 324(5934), 1557-1561. https://doi.org/10.1126/scien ce. 1176062.

6. Liu, Y., Gayle, A. A., Wilder-Smith, A., \& Rocklov, J. (2020). The reproductive number of COVID-19 is higher compared to SARS coronavirus. Journal of Travel Medicine. https://doi.org/10.1093/ jtm/taaa021.

7. Biggerstaff, M., Cauchemez, S., Reed, C., Gambhir, M., \& Finelli, L. (2014). Estimates of the reproduction number for seasonal, pandemic, and zoonotic influenza: A systematic review of the literature. BMC Infectious Diseases, 14(1), 480. https:// doi.org/10.1186/1471-2334-14-480.

8. Ajilore, K., Atakiti, I., \& Onyenankeya, K. (2017). College students' knowledge, attitudes and adherence to public service announcements on Ebola in Nigeria: Suggestions for improving future Ebola prevention education programmes. Health Education Journal, 76(6), 648-660. https://doi.org/10.1177/00178 96917710969.

9. Tachfouti, N., Slama, K., Berraho, M., \& Nejjari, C. (2012). The impact of knowledge and attitudes on adherence to tuberculosis treatment: A case-control study in a Moroccan region. The Pan African Medical Journal, 12, 52-52.

10. Babiker, A., El Husseini, M., Al Nemri, A., et al. (2014). Health care professional development: Working as a team to improve patient care. Sudanese Journal of Paediatrics, 14(2), 9-16.

11. Askarian, M., Danaei, M., \& Vakili, V. (2013). Knowledge, attitudes, and practices regarding pandemic H1N1 influenza among medical and dental residents and fellowships in Shiraz Iran. International Journal of Preventive Medicine, 4(4), 396-403.

12. Khowaja, Z. A., Soomro, M. I., Pirzada, A. K., Yoosuf, M. A., \& Kumar, V. (2011). Awareness of the pandemic H1N1 influenza global outbreak 2009 among medical students in Karachi Pakistan. Journal of Infection in Developing Countries, 5(3), 151-155. https://doi.org/10.3855/jidc. 1247.

13. Scott, L. A., Carson, D. S., \& Greenwell, I. B. (2010). Disaster 101: A novel approach to disaster medicine training for health professionals. Journal of Emergency Medicine, 39(2), 220-226. https://doi.org/10.1016/j.jemermed.2009.08.064.

14. Zhong, B.-L., Luo, W., Li, H.-M., et al. (2020). Knowledge, attitudes, and practices towards COVID-19 among Chinese residents during the rapid rise period of the COVID-19 outbreak: A quick online cross-sectional survey. International Journal of Biological Sciences, 16(10), 1745-1752. https://doi. org/10.7150/ijbs.45221.

15. Taber, K. S. (2018). The use of cronbach's alpha when developing and reporting research instruments in science education. Research in Science Education, 48(6), 1273-1296. https://doi. org/10.1007/s11165-016-9602-2.

16. Dhimal, M., Aryal, K. K., Dhimal, M. L., et al. (2014). Knowledge, attitude and practice regarding dengue fever among the healthy population of highland and lowland communities in central Nepal. PLoS ONE, 9(7), e102028. https://doi.org/10.1371/ journal.pone.0102028.

17. Chen, N., Zhou, M., Dong, X., et al. (2020). Epidemiological and clinical characteristics of 99 cases of 2019 novel coronavirus pneumonia in Wuhan, China: A descriptive study. The Lancet, 395(10223), 507-513. https://doi.org/10.1016/S0140 -6736(20)30211-7.

18. Hedima, E. W., Adeyemi, M. S., \& Ikunaiye, N. Y. (2020). Community pharmacists: On the frontline of health service against COVID-19 in LMICs. Research in Social \& Administrative Pharmacy: RSAP. https://doi.org/10.1016/j.sapha rm.2020.04.013.

19. Khan, M. U., Shah, S., Ahmad, A., \& Fatokun, O. (2014). Knowledge and attitude of healthcare workers about middle east respiratory syndrome in multispecialty hospitals of Qassim Saudi Arabia. BMC Public Health, 14, 1281. https://doi. org/10.1186/1471-2458-14-1281.

20. Hoda, J. (2016). Identification of information types and sources by the public for promoting awareness of middle east respiratory syndrome coronavirus in Saudi Arabia. Health Education Research, 31(1), 12-23. https://doi.org/10.1093/her/cyv061.

21. Abdelhafiz, A. S., Mohammed, Z., Ibrahim, M. E., et al. (2020). Knowledge, perceptions, and attitude of Egyptians towards the novel coronavirus disease (COVID-19). Journal of Community Health. https://doi.org/10.1007/s10900-020-00827-7. 
22. Li, L. Q., \& Huang, T. (2020). COVID-19 patients' clinical characteristics, discharge rate, and fatality rate of meta-analysis. Journal of Medical Virology. https://doi.org/10.1002/jmv.25757.

23. Tang, N., Bai, H., Chen, X., Gong, J., Li, D., \& Sun, Z. (2020). Anticoagulant treatment is associated with decreased mortality in severe coronavirus disease 2019 patients with coagulopathy. Journal of Thrombosis and Haemostasis. https://doi.org/10.1111/ jth.14817.

24. Guan, W. J., Ni, Z. Y., Hu, Y., et al. (2020). Clinical characteristics of coronavirus disease 2019 in China. The New England Journal of Medicine. https://doi.org/10.1056/NEJMoa2002032.

25. Feng, S., Shen, C., Xia, N., Song, W., Fan, M., \& Cowling, B. J. (2020). Rational use of face masks in the COVID-19 pandemic.
The Lancet Respiratory Medicine, 8(5), 434-436. https://doi. org/10.1016/s2213-2600(20)30134-x.

26. McCloskey, B., Zumla, A., Ippolito, G., et al. (2020). Mass gathering events and reducing further global spread of COVID-19: A political and public health dilemma. The Lancet, 395(10230), 1096-1099. https://doi.org/10.1016/S0140-6736(20)30681-4.

Publisher's Note Springer Nature remains neutral with regard to jurisdictional claims in published maps and institutional affiliations. 